\title{
I3S: Intelligent spectrum sensing scheme for cognitive radio networks
}

\author{
Waleed Ejaz ${ }^{1}$, Najam ul Hasan', Seok Lee ${ }^{2}$ and Hyung Seok Kim ${ }^{1 *}$
}

\begin{abstract}
Reliable spectrum sensing is one of the most crucial aspects for the successful deployment of cognitive radio (CR) technology. For CR, it is not possible to transmit on a licensed band and sense it simultaneously, therefore sensing must be interleaved with transmission. Spectrum sensing in CR is challenged by a number of uncertainties, which degrade the sensing performance and in turn require much more time to achieve the targeted sensing efficiency. Hence, the authors propose a spectrum sensing scheme which obtains reliable results with less mean detection time. First, the scheme determines a better matched filter, or a combination of energy and cyclostationary detectors based on the power and band of interest. In the combined energy and cyclostationary detector, an energy detector with a bi-threshold is used, and the cyclostationary detector is applied only if the energy of the signal lies between two thresholds. Second, sensing is performed by the selection choice resulting from the first step. To evaluate the scheme's performance, the results are compared with those where only an energy detector, matched filter, or cyclostationary detector are performed. The performance metrics are the probability of detection, probability of false alarm, and mean detection time.
\end{abstract}

Keywords: Cognitive radio networks, Spectrum sensing, Energy detection, Cyclostationary detection, Matched filter

\section{Introduction}

Technologies for wireless communication have advanced in recent years. The demand for radio spectrum increases proportionally with the number of users, and thus causes a significant increase in spectrum utilization. The major hurdle in the current spectrum scarcity is the fixed spectrum assignment. This spectrum shortage has a deep impact on research directions in the field of wireless communication.

Cognitive radio (CR) is a key technology for dealing with the current underutilization of spectrum [1]. The CR network allows CR users/secondary users (SUs) to access a spectrum which is not in use by a licensed user/primary user (PU). The most essential task of a CR network is to detect the presence or absence of a PU in order for the SU to use the licensed band efficiently and to avoid interference in the PU vicinity. The process of PU detection is called spectrum sensing. Currently, spectrum sensing techniques focus on PU transmitter detection. The local

\footnotetext{
*Correspondence: hyungkim@sejong.edu

1 Department of Information \& Communication Engineering, Sejong University, Seoul, Republic of Korea

Full list of author information is available at the end of the article
}

sensing techniques considered to be important are energy detection, matched filter detection, and cyclostationary detection [2]. Energy detection needs less sensing time but performs poorly under low signal-to-noise ratio (SNR) conditions. One of the well-known coherent detection techniques in the field of spectrum sensing is matched filter detection. Cyclostationary detection provides reliable detection but is computationally complex.

The probability of detection $\left(P_{d}\right)$ and the probability of false alarm $\left(P_{f}\right)$ are the metrics for the detection performance of spectrum sensing. The probability that an $\mathrm{SU}$ declares the presence of a PU when the spectrum is occupied by the PU is called the probability of detection, whereas the probability that an SU declares the presence of the PU when the spectrum is idle is called the probability of false alarm. The probability of miss detection $\left(P_{m}\right)$ indicates the probability that an SU declares the absence of a PU when the spectrum is occupied. The probability of miss detection is simply, $P_{m}=1-P_{d}$. In view of the fact that false alarms reduce spectral efficiency and miss detection causes interference with the PU, generally it is vital for optimal detection performance so that the

\section{黛 Springer}

(c) 2013 Ejaz et al.: licensee Springer. This is an Open Access article distributed under the terms of the Creative Commons Attribution License (http://creativecommons.org/licenses/by/2.0), which permits unrestricted use, distribution, and reproduction in any medium, provided the original work is properly cited. 
maximum probability of detection is achieved subject to the minimum probability of false alarm [3].

The matched filter is optimal if structure of PU waveform is known. If deployment of CR is limited to operate in few PU bands then matched filer is the best choice. However, the implementation cost and complexity will increase if more PU bands are considered because dedicated circuitry is required for each primary licensee to achieve synchronization [4]. Practically, it is not possible to devote circuitry for each PU licensee. However, matched filter can be considered for most frequent sensed channels to get optimal sensing results with minimum sensing time if $\mathrm{PU}$ waveform is known. This approach can be very healthy for $C R$ applications for disaster management; smart grid, and so on to get reliable sensing results with minimum sensing time. Many improved local sensing schemes are proposed in [5-12], including our own fuzzy logic-based and SNR-based adaptive spectrum sensing for improved local sensing. In the proposed scheme, channels with known PU waveform will be sensed by matched filter detection and rest of the channels by the detectors which do not need dedicated circuitry and prior knowledge of PU waveform.

In this article, we propose an intelligent spectrum sensing scheme(I3S) based on the energy detection, matched filter detection, and cyclostationary detection. It is assumed that a CR network has to detect multiple PU systems and that the PU waveform for some of the PU systems is known. The SU analyzes based on the power and the band of interest regardless of whether the PU waveform is known or not. The SU then performs either the combination of energy detection and cyclostationary detection if the PU waveform is unknown, or matched filter detection if the PU waveform is known. The performance of the I3S is analyzed in terms of the probability of detection, the probability of false alarm, and the mean detection time to determine the occupancy of a channel.

This study is different from all existing improvements in two ways:

- The proposed scheme intelligently decides the detection algorithm based on the power and band of interest, thus increasing accuracy and reducing mean detection time for the known PU waveforms. According to the author's best knowledge, none of the existing approaches have incorporated the information of the band for the selection of the detector.

- All other schemes consider multiple detectors working sequentially.

The remainder of this article is organized as follows. In Section 2, various improved local spectrum sensing schemes are briefly discussed. Section 3 presents the system model and framework. Section 4 analyzes the scheme from the viewpoint of detection performance and mean detection time. In Section 5, we present simulation results and their detailed analysis, and finally, conclusions are drawn in Section 6.

\section{Related work}

Spectrum sensing is fundamental for the successful deployment of CRs. The main focus of current spectrum sensing schemes for $\mathrm{CRs}$ is divided into two main streams: the first is to improve local sensing performance, and the second is to improve performance by having cooperation between SUs. In local sensing, each SU performs spectrum sensing on the received signal and makes a decision about the presence or absence of a PU. In cooperative spectrum sensing, SUs perform local sensing and send their sensed information to the fusion center, and a final cooperative decision is taken at the fusion center. Therefore, in order to improve cooperative performance, it is necessary to improve local sensing. Many two-stage spectrum sensing schemes are proposed in literature to improve local spectrum sensing.

In [5], a two-stage fuzzy logic-based detection (FLD) scheme is proposed. In the first stage, each CR performs existing spectrum sensing techniques, i.e., energy detection, matched filter detection, and cyclostationary detection. While in the second stage, the output from each technique employed in the first stage is combined using fuzzy logic to ultimately decide about the presence or absence of a PU.

A low power discrete Fourier transform (DFT) filter bank-based two-stage spectrum sensing is proposed in [6]. Energy detector is used for the first stage course sensing and then in the second stage fine sensing it is complemented by the cyclostationary detection. Authors exploited the fact that power of sensing operation depends on the sampling rate. Therefore, polyphase DFT filter bank is used to choose appropriate sampling rate.

SNR-based two-stage adaptive spectrum sensing is proposed in [7]. In the first stage, the SNR is estimated in advance for available channels. The SU then performs either energy detection or cyclostationary detection based on the SNR estimated in the first stage of PU detection.

A novel high-speed two-stage detector is proposed in [8] that effectively decreased the sensing time by satisfying the required detection capabilities. Energy detector is used in the coarse sensing stage and if the measured energy is greater than threshold then it declares PU present, else it computes the SNR of device. If the computed SNR is greater than theoretical SNR, then the result of energy detector is reliable. If computed SNR is less than theoretical SNR then second stage for fine sensing is performed in which covariance absolute value is used. 
In [9], another two-stage sensing scheme is proposed in which, at the first stage, the energy detector is used, and if required, cyclostationary detection is used at the second stage. The second stage will run only if a channel is declared unoccupied in the first stage. In this case, the second stage will give a final decision about the presence or absence of a PU. If a channel is declared occupied, the first stage will provide the final decision.

An improved version of [9] in terms of mean detection time is proposed in [10]. It achieves the same probability of detection and false alarm with much less mean detection time. The first stage will run in the same way as previously discussed, but before the second stage, it estimates the SNR of the received signal and determines the credibility of the energy detector. If the energy detector is credible, it declares the absence of a PU at the first stage, otherwise it will run the second stage in order to get an accurate decision about the presence or absence of a PU.

A two-stage spectrum sensing scheme is also proposed in [11], in which the energy detector is used at the first stage to sort channels in ascending order based on the power of each channel. The one-order cyclostationary detector is used on the channel with the lowest power to detect weak signals in the second stage.

A two-stage dynamic spectrum access approach, which consists of preliminary coarse resolution sensing (CRS) followed by fine resolution sensing (FRS), is proposed in [12]. In CRS, the whole spectrum is divided into equalsized coarse sensing blocks (CSB) of equal bandwidth, and an energy detector of bandwidth equal to that of the CSB is applied on randomly selected CSB and checked for at least one idle channel. FRS is then applied on the same $\mathrm{CSB}$, using the energy detector equal to the bandwidth of the channel to determine its unused channel.

In our proposed scheme, based on the power and band of interest, we first determine information about the PU waveform. The distinction of the proposed scheme is that it deals with multiple types of primary systems, i.e., for primary systems with known and unknown waveforms. Whereas all the existing two-stage detection schemes in the literature only considered single type of primary system.

\section{System model and framework}

A binary hypothesis model for transmitter detection, i.e., the model of signals received by the SU, is defined as

$$
r(t)= \begin{cases}n(t), & \text { if } H_{0} \\ n(t)+h s(t), & \text { if } H_{1}\end{cases}
$$

where $r(t)$ is the signal received by the CR, $s(t)$ is the transmitted signal of the PU, $n(t)$ is additive white Gaussian noise (AWGN), and $h$ is the amplitude gain of the channel. $H_{0}$ indicates only noise, and $H_{1}$ indicates the presence of PU.

The proposed I3S system model is shown in Figure 1 and description of modeling variables is given in Table 1. Multiple PU systems are considered for detection. It is assumed that, for some PU systems, the signal structure is unknown, while for the others, enough information is known about the PU waveform to perform the matched filter as an optimal detector. It is assumed that there are $N$ channels to be sensed. The SU will scan the whole spectrum and detect whether or not there is a spectrum hole available. The SU identifies that a PU waveform is known (or not) on the basis of the power and band of interest, and then selects either a combined energy and cyclostationary detector, or a matched filter detector.

\subsection{Combined energy and cyclostationary detector}

If the PU waveform is unknown, the energy detector is applied on the received signal $r(t)$. An energy detector with bi-thresholds is used for detection in which two thresholds $\lambda_{1}$ and $\lambda_{2}$ are used. The received energy is given by

$$
E=\sum_{k=0}^{j-1}|r(k)|^{2}
$$

where $j$ is determined from the time bandwidth product. If the received energy $E$ is greater than $\lambda_{1}$, then the presence of a PU is declared. Similarly, if the received signal is less than $\lambda_{2}$, then the absence of a PU is declared. If the received signal energy is between $\lambda_{1}$ and $\lambda_{2}$, it is in the region of uncertainty (RU), and the energy detector is not reliable for PU detection, which is evaluated as

$$
\text { Decision }= \begin{cases}1, & \text { if } E>\lambda_{1} \\ \mathrm{RU}, & \text { if } \lambda_{2} \leq E \leq \lambda_{1} \\ 0, & \text { if } E<\lambda_{2}\end{cases}
$$

The output $E$ of the energy detector has a distribution that is defined as follows [13].

$$
E= \begin{cases}\chi_{2 w}^{2}, & \text { if } H_{0} \\ \chi_{2 w}^{2}(2 \gamma), & \text { if } H_{1}\end{cases}
$$

where $\chi_{2 w}^{2}$ and $\chi_{2 w}^{2}(2 \gamma)$ represent a central chi-square distribution and a non-central chi-square distribution with $2 w$ degrees of freedom and the non-centrality parameter $2 \gamma$, respectively.

The approximation for the probability of detection $P_{d, E}$ and probability of false alarm $P_{f, E}$ of the energy detector 


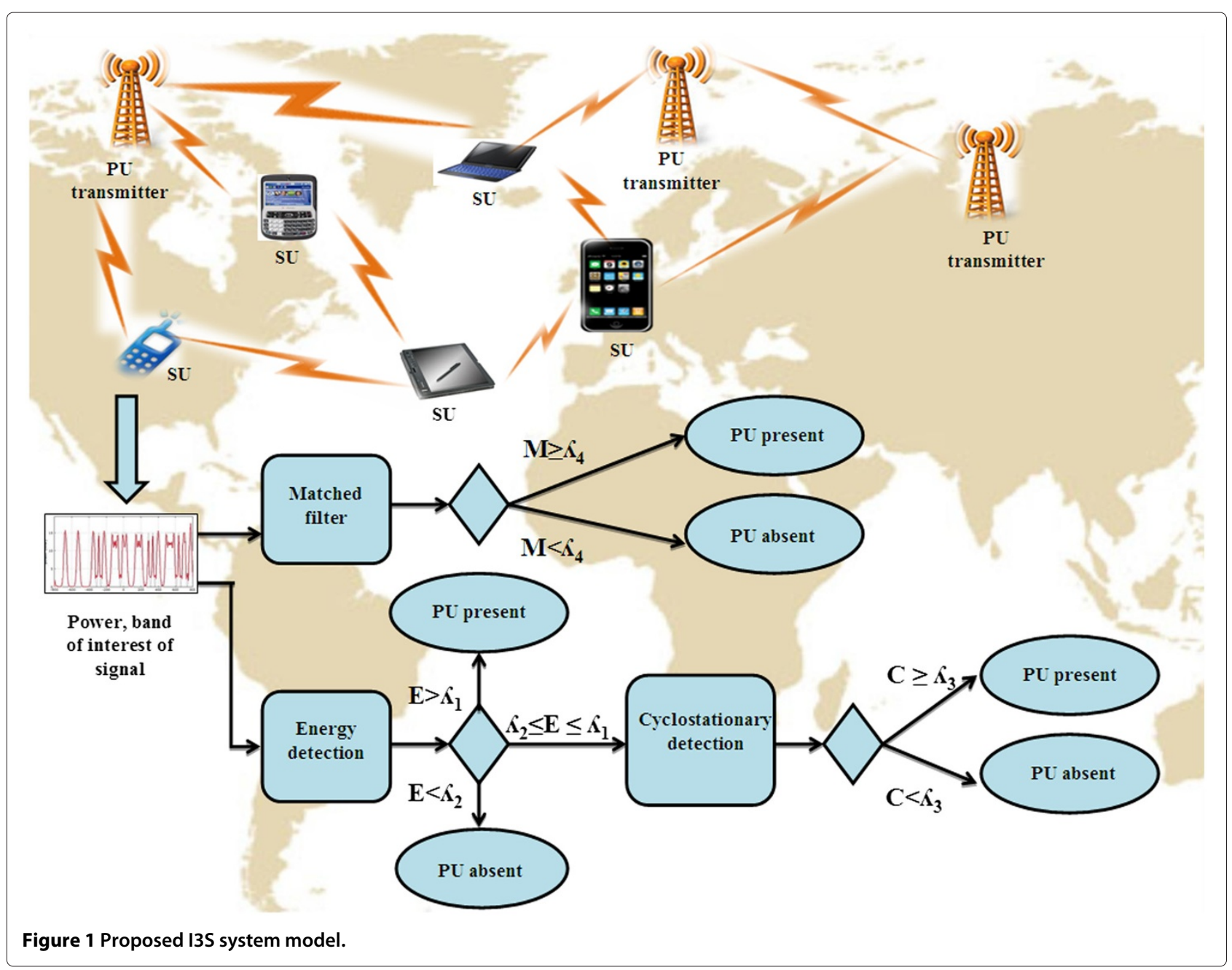

with bi-thresholds over the AWGN channel can be given by [9]:

$$
\begin{aligned}
& P_{d, E}=Q_{m}\left(\sqrt{\frac{2 \gamma}{\delta^{2}}}, \sqrt{\frac{\lambda_{2}}{\delta^{2}}}\right) \\
& P_{f, E}=\frac{\Gamma\left(m, \lambda_{1} / 2 \delta^{2}\right)}{\Gamma(m)}
\end{aligned}
$$

where $\Gamma($.$) and \Gamma(.,$.$) are complete and incomplete$ gamma functions, respectively. $Q_{m}(.,$.$) is the general-$ ized Marcum $Q$-function, $\gamma$ is the instantaneous SNR, $\delta^{2}$ is the noise variance and the time bandwidth product is assumed to be an integer number denoted by $m$.

Therefore, the cyclostationary detector is applied for a reliable decision of sensing accuracy. Researchers suggest that cyclostationary feature detection is more suitable than the energy detector technique when the noise uncertainties are unknown [2]. Although the energy detector, as a non-coherent detection method, does not require any prior knowledge of a PU's waveform and so is easy to implement, it is highly susceptible to in-band interference and changing noise levels [14], and cannot differentiate between signal and noise power.

Commonly, the primary modulated waveforms are coupled with patterns also characterized as cyclostationary features, like sine wave carriers, pulse trains, repeating spreading, hopping sequences, and cyclic prefixes inducing periodicity [15]. An SU can detect a random signal with a specific modulation type in the presence of random stochastic noise by exploiting periodic statistics like the mean and auto-correlation of a PU waveform. Features like autocorrelation and mean are estimated by analyzing spectral correlation functions (SCFs). A block diagram of cyclostationary detection using the SCF is shown in Figure 2.

The SCF, also called a cyclic spectrum, is a twodimensional function with a cyclic frequency $\alpha$. Power spectral density is a special case of the SCF with $\alpha=0$. The detected features are the number of signals, 
Table 1 Description of modeling variables

\begin{tabular}{|c|c|}
\hline Symbol & Description \\
\hline N & Number of channels to be sensed \\
\hline$P_{d}$ & Probability of detection of I3S scheme \\
\hline$P_{f}$ & Probability of false alarm of I3S scheme \\
\hline$P_{m}$ & Probability of miss detection of I3S scheme \\
\hline$r(t)$ & Received signal \\
\hline$s(t)$ & Transmitted signal \\
\hline$n(t)$ & Additive white gaussian noise (AWGN) \\
\hline$h$ & Amplitude gain of the channel \\
\hline$H_{0}$ & Indicates absence of PU \\
\hline$H_{1}$ & Indicates presence of PU \\
\hline E & Received energy at output of energy detector \\
\hline$\gamma$ & Instantaneous SNR \\
\hline$m$ & time bandwidth product \\
\hline$P_{d, \mathrm{E}}$ & Probability of detection for bi-threshold energy detector \\
\hline$P_{f, \mathrm{E}}$ & Probability of false alarm for bi-threshold energy detector \\
\hline$P_{d, C}$ & Probability of detection for cyclostationary detector \\
\hline$P_{f, C}$ & Probability of false alarm for cyclostationary detector \\
\hline$P_{d, \mathrm{EC}}$ & $\begin{array}{l}\text { Probability of detection for combined energy and cyclo- } \\
\text { stationary detector }\end{array}$ \\
\hline$P_{f, \mathrm{EC}}$ & $\begin{array}{l}\text { Probability of false alarm for combined energy and cyclo- } \\
\text { stationary detector }\end{array}$ \\
\hline$P_{d, M}$ & Probability of detection for matched filter \\
\hline$P_{f, M}$ & Probability of false alarm for matched filter \\
\hline P & $\begin{array}{l}\text { Probability that channels are sensed by cyclostationary } \\
\text { detector }\end{array}$ \\
\hline$P_{r}$ & $\begin{array}{l}\text { Probability that channel would sensed by combined } \\
\text { energy and cyclostationary detector }\end{array}$ \\
\hline $1-P_{r}$ & Probability that channel would sensed by matched filter \\
\hline $\bar{T}$ & Mean detection time of I3S scheme \\
\hline$\overline{T_{\mathrm{EC}}}$ & $\begin{array}{l}\text { Mean detection time of combined energy and cyclosta- } \\
\text { tionary detector }\end{array}$ \\
\hline$\overline{T_{M}}$ & Mean detection time of matched filter \\
\hline$\overline{T_{E}}$ & Mean detection time of energy detector \\
\hline$\overline{T_{C}}$ & Mean detection time of cyclostationary detector \\
\hline
\end{tabular}

their modulation types, symbol rates, and presence of interferers. Using the computed SCF and a hypothesis model for spectrum sensing, we can determine whether or not a signal of a specific cyclic frequency of interest is present [16].
The probability of detection $P_{d, C}$ and the probability of false alarm $P_{f, C}$ for a cyclostationary detector over the AWGN channel are given by [17]

$$
\begin{aligned}
& P_{d, C}=1-\left[1-Q_{m}\left(\frac{2 \gamma}{\sigma_{w}}, \frac{\lambda_{3}}{\delta_{A}}\right)\right]^{L} \\
& P_{f, C}=1-\left(1-e^{-\frac{\lambda_{3}}{2 \delta_{A}^{2}}}\right)^{L}
\end{aligned}
$$

where $\sigma_{w}^{2}$ is the variance, $\delta_{A}^{2}=\sigma_{w}^{2} /\left(2 M_{c}+1\right)$ in which $M_{c}$ is the number of samples for detection, $L$ is the number of diversity branches, $\gamma$ is instantaneous $\operatorname{SNR}, Q_{m}(.,$.$) is$ the generalized Marcum $Q$-function, and $\lambda_{3}$ is a predetermined threshold. The decision metric $C$ is compared with $\lambda_{3}$ for PU presence or absence.

The overall probability of detection and false alarm of the combined energy and cyclostationary detector is given as

$$
\begin{aligned}
& P_{d, \mathrm{EC}}=P_{d, \mathrm{E}}-P_{0}\left(1-P_{d, \mathrm{C}}\right) \\
& P_{f, \mathrm{EC}}=P_{f, \mathrm{E}}-P_{1}\left(1-P_{f, \mathrm{C}}\right)
\end{aligned}
$$

where $P_{0}$ is the probability that the received energy is between $\lambda_{1}$ and $\lambda_{2}$ when a PU is present, and $P_{1}$ is the probability that the received energy is between $\lambda_{1}$ and $\lambda_{2}$ when a PU is absent. In the I3S, when the received energy is between $\lambda_{1}$ and $\lambda_{2}$, channels are sensed by the cyclostationary detector. Thus, $P=P_{1}+P_{2}$ is the same as the probability that channels are sensed by the cyclostationary detector.

\subsection{Matched filter detection}

One of the well-known techniques of spectrum sensing for a known PU waveform is matched filter detection. The intuition behind the matched filter relies on the prior knowledge of a PU waveform, such as modulation type, order, the pulse shape, and the packet format. To meet such a stringent condition, CRs need to have a cache for the pattern information in their memory and to satisfy synchronization. The matched filter is an optimal linear filter for maximizing the SNR in the presence of additive stochastic noise [18]. The matched filter is equivalent to convolving the received signal $r(t)$ with a time-reversed version of the known signal or template as

$$
r(t) * s(T-t+\tau)
$$

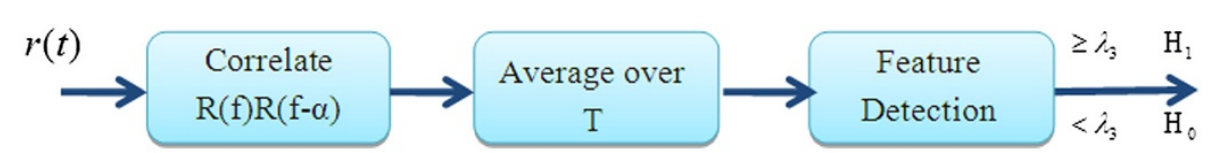

Figure 2 Block diagram of cyclostationary detection. 
where $T$ is a symbol time duration and $\tau$ is the shift in the known signal. Finally, the output of matched filter $M$ is compared with a threshold factor $\lambda_{4}$ to decide about the presence or absence of a PU as shown in Figure 3. The probability of detection $P_{d, M}$ and the probability of false alarm $P_{f, M}$ of the matched filter are given [19] as

$$
\begin{gathered}
P_{d, M}=Q\left(\frac{\lambda_{4}-E}{\sigma_{w} \sqrt{E}}\right) \\
P_{f, M}=Q\left(\frac{\lambda_{4}}{\sigma_{w} \sqrt{E}}\right)
\end{gathered}
$$

where $Q($.) is the Gaussian complexity distribution function, $E$ is the energy of the deterministic signal of interest, and $\sigma_{w}^{2}$ is the noise variance.

\section{Analysis of sensing performance}

The sensing performance of the proposed I3S is analyzed with respect to the probability of detection and false alarm. The overall probability of detection and the probability of false alarm of the proposed scheme are given as

$$
\begin{gathered}
P_{d}=P_{r} P_{d, E C}+\left(1-P_{r}\right) P_{d, M} \\
P_{f}=P_{r} P_{f, E C}+\left(1-P_{r}\right) P_{f, M}
\end{gathered}
$$

where $P_{r}$ is the probability that a channel would be sensed using the combined energy and cyclostationary detector. Therefore, the probability that a channel would be sensed by the matched filter detection will be $1-P_{r} . P_{r}$ is dependent on the power and band of interest of the channels to be sensed.

In order to evaluate the agility of the I3S, its mean detection time is compared with the energy detection, matched filter detection, and cyclostationary detection. It is assumed that there is a total of $N$ channels, and $L$ out of $N$ channels are sensed by the combined energy and cyclostationary detector. The mean detection time of the proposed sensing scheme is

$$
\bar{T}=\bar{T}_{\mathrm{EC}}+\bar{T}_{\mathrm{M}}
$$

where $\bar{T}_{\text {EC }}$ and $\bar{T}_{\mathrm{M}}$ are the sensing times of the combined energy and cyclostationary detector and the matched filter detection, respectively. $\bar{T}_{\mathrm{EC}}$ is

$$
\bar{T}_{\mathrm{EC}}=\bar{T}_{\mathrm{E}}+\bar{T}_{\mathrm{C}}
$$

where $\bar{T}_{\mathrm{E}}$ and $\bar{T}_{\mathrm{C}}$ are the sensing times of energy detection and cyclostationary detection, respectively. $\bar{T}_{\mathrm{E}}$ and $\bar{T}_{\mathrm{C}}$ can be derived as follows

$$
\bar{T}_{\mathrm{E}}=N P_{r} T_{1}
$$

where $T_{1}=\frac{M_{\mathrm{E}}}{2 W}$ is the mean sensing time for each channel, in which $M_{\mathrm{E}}$ is the number of samples during the observation interval, and $W$ is the channel bandwidth. $\bar{T}_{\mathrm{C}}$ can be derived as follows

$$
\bar{T}_{\mathrm{C}}=E\left[K_{1}\right] T_{2}
$$

where $E\left[K_{1}\right]$ represents the mean number of channels sensed by the cyclostationary detector and $T_{2}=\frac{M_{C}}{2 W}$ is the mean sensing time for each channel in which $M_{C}$ is the number of samples for detection, and $W$ is the channel bandwidth. $K_{1}$ is a random variable which follows a binomial distribution, with parameters $N$ and $P$, where $N$ is the number of sensed channels and $P$ is the probability that a channel will be sensed by the cyclostationary detector. Hence, the mean detection time of the cyclostationary detector is

$$
\overline{T_{\mathrm{C}}}=N P_{r} P T_{2}
$$

The channels are sensed by the combined energy and cyclostationary detector with the probability $P_{r}$. The total mean detection time is found by substituting (18) and (20) for $\bar{T}_{\mathrm{E}}$ and $\bar{T}_{\mathrm{C}}$ in (17)

$$
\bar{T}_{\mathrm{EC}}=N P_{r}\left(T_{1}+P T_{2}\right) .
$$

$\bar{T}_{\mathrm{M}}$ is derived as follows

$$
\bar{T}_{\mathrm{M}}=E\left[K_{2}\right] T_{3}
$$

where $E\left[K_{2}\right]$ represents the mean number of channels sensed by the matched filter detection, and $T_{3}$ is the mean sensing time for each channel. $K_{2}$ is a random variable which follows a binomial distribution, with parameters $N$ and $\left(1-P_{r}\right)$, where $N$ is the number of sensed channels, and $\left(1-P_{r}\right)$ is the probability that a channel will be sensed by the matched filter detection. Hence, the mean detection time of the matched filter detection is

$$
\bar{T}_{\mathrm{M}}=N\left(1-P_{r}\right) T_{3} \text {. }
$$

The overall mean detection time of the proposed I3S from (16) is

$$
\bar{T}=N\left[P_{r}\left(T_{1}+P T_{2}\right)+\left(1-P_{r}\right) T_{3}\right] .
$$

We can make the following two cases on the basis of $P_{r}$ and $P$.

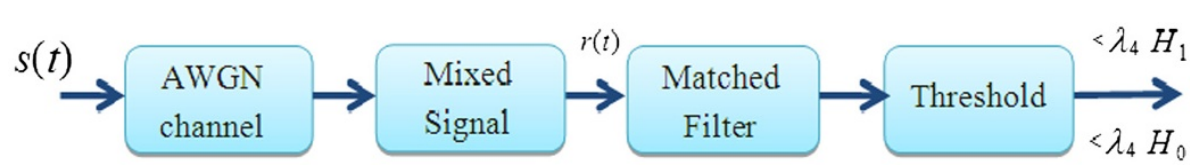

Figure 3 Block diagram of matched filter. 
Case 1: When $0 \leq P_{r}<0.5$ for the majority of the channels, the PU waveform is known. Therefore, the SU will perform matched filter detection for sensing most of the channels because the performance of the matched filter is optimal when the PU waveform is known. The mean detection time of the matched filter detection is the least, and therefore the best case for the detection time is when the majority of channels are sensed by the matched filter. The best scenario is when $P_{r} \approx 0$, and the probability of detection, the probability of false alarm, and the mean detection time can be found by putting $P_{r} \approx 0$ and $P \approx 0$ in (14), (15), and (24), respectively:

$$
\begin{aligned}
P_{d} & \approx P_{d, M} \\
P_{f} & \approx P_{f, M} \\
\bar{T} & \approx N T_{3}
\end{aligned}
$$

Case 2: When $0.5<P_{r} \leq 1$ for most of the channels, the PU waveform is unknown. The SU will perform combined energy and cyclostationary detector for sensing the majority of channels. The detection time will increase when more channels are sensed by the cyclostationary detector, i.e., higher value of $P$. In the worst case, the probability of detection, the probability of false alarm, and the mean detection time can be evaluated by putting $P_{r} \approx 1$ and $P \approx 1$ in (14), (15), and (24):

$$
\begin{aligned}
& P_{d} \approx P_{d, \mathrm{EC}} \\
& P_{f} \approx P_{f, \mathrm{EC}} \\
& \bar{T} \approx N\left[\left(T_{1}+T_{2}\right)\right]
\end{aligned}
$$

\section{Simulation results}

In this section, we compare the I3S with matched filter detection, energy detection, and cyclostationary detection. The sensing performance of each detection scheme is quantified by the receiver operating characteristic (ROC), such as $P_{f}$ versus $P_{d}$ and the mean sensing time. Monte Carlo simulation was used for the experimentation under the following system settings: there are 10 randomly distributed Gaussian channels with zero mean and variance 1 , and an SU looking for spectrum holes in these channels. Figure 4 shows the probability of detection versus the average SNR, when the probability that channels the combined energy and cyclostationary detector goes to the cyclostationary stage, changes from 0.9 to 0.1 with a step of -0.4 . The probability $P_{r}$ that the channels are sensed by the combined energy and cyclostationary detector is fixed at 0.8 . A change in $P$ means that the thresholds $\lambda_{1}$ and $\lambda_{2}$ of the bi-threshold energy detector change. Increasing $\lambda_{1}$ and decreasing $\lambda_{2}$ will increase the overall probability of detection at the cost of increased detection time. In this sense, Figure 4 can serve as a guideline for network

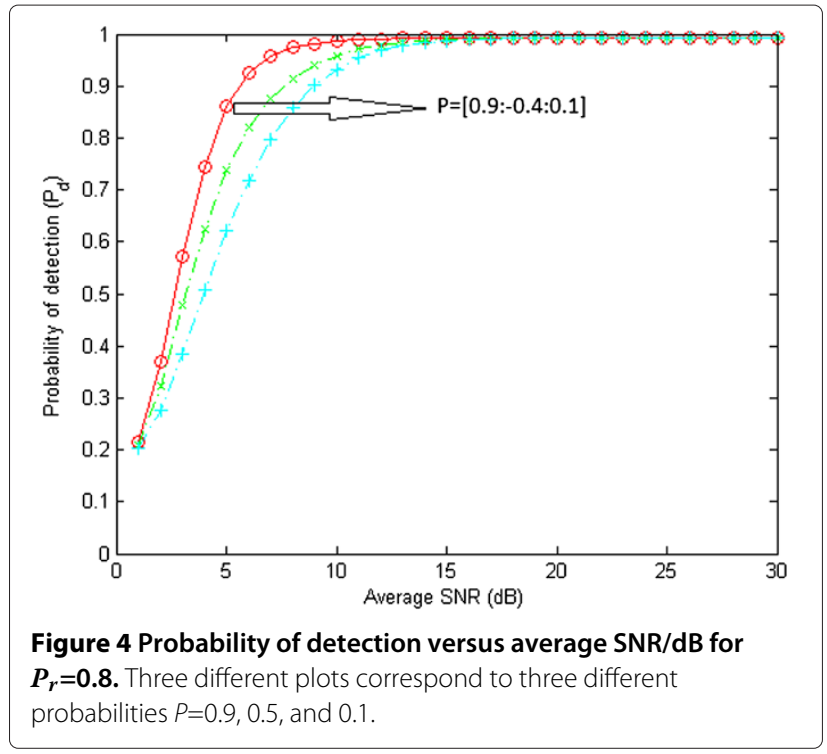

designers to set optimal values of thresholds $\lambda_{1}$ and $\lambda_{2}$ for the desired probability of detection.

In Figure 5, the probability of miss detection versus average SNR is presented for the probability of false alarm as 0.1 and 0.01 , respectively. The results are conducted for $P_{r}=0.8$ which means that most of the channels are sensed by the combined energy and cyclostationary detector. $P$ is taken to be 0.5 , which means that half of the channels detected by the combined energy and cyclostationary detector need to go through the cyclostationary detector. The result shows that the probability of miss detection is decreased with the increase of average SNR. For a specific value of average SNR, high $P_{f}$ results in a low probability of miss detection because of the decreased threshold.

Figure 6 shows the ROCs of the I3S, the matched filter detection, the energy detection, and the cyclostationary

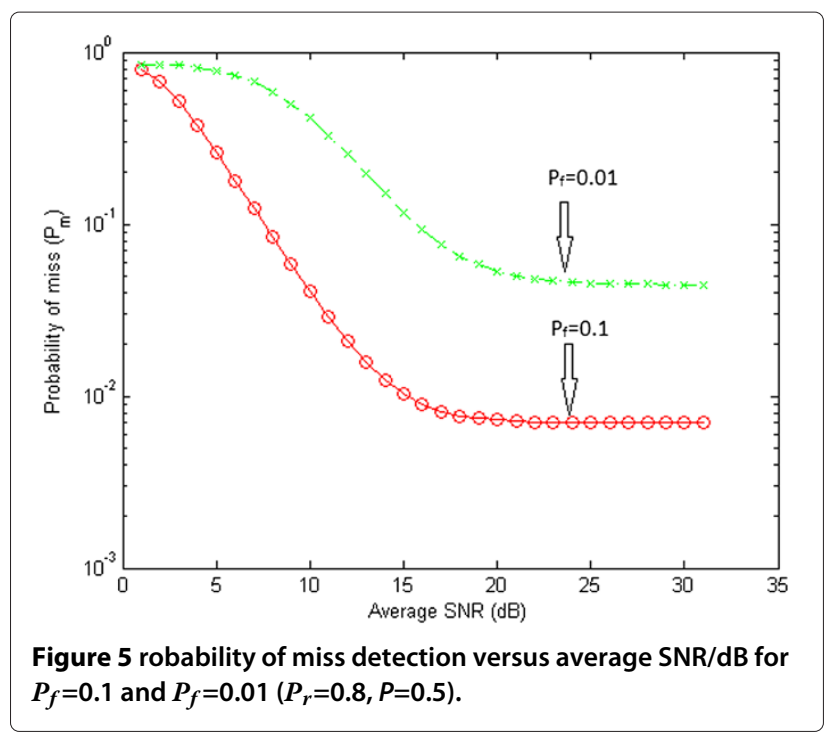




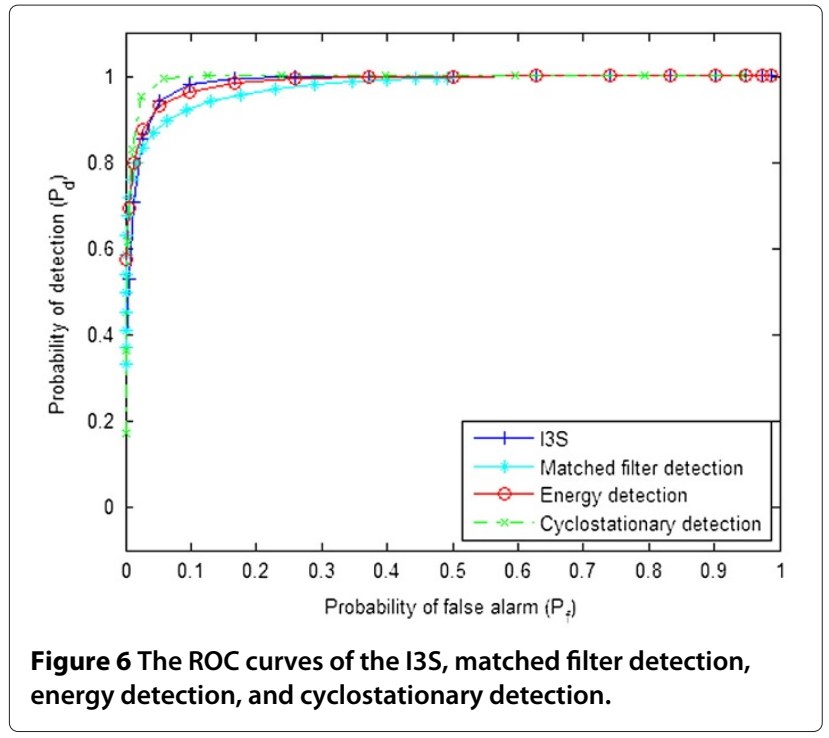

detection. In this scenario, it is assumed that the average SNR is $-10 d B$. Both $P_{r}$ and $P$ are taken to be 0.5 . The result shows that the proposed scheme performs as well as cyclostationary detection for $P_{f}$ greater than $10^{-1}$.

The mean detection time of the I3S, matched filter detection, energy detection, and cyclostationary detection is evaluated with varying $P_{r}$ in Figure 7. The mean detection time of all the detection schemes remains constant regardless of $P_{r}$, except for the I3S. If $P_{r}$ is low, most of the channels are sensed by the matched filter detection, and the mean detection time is almost equal to the time taken by matched filter detection. Figure $7 \mathrm{a}-\mathrm{c}$ shows the graph of the mean detection time with varying $P_{r}$ from 0 to 1 , and $P$ is taken as $0.2,0.5$, and 0.8 , respectively. When $P_{r}$ is high, the I3S does not always have a smaller mean detection time in comparison with the energy detection and matched filter detection because the majority of channels are sensed by the combined energy and cyclostationary detector. When $P$ is 0.2 , most of the channels sensed by the combined energy and cyclostationary detector are concluded at the energy detector stage. The mean detection time of the I3S is much less in comparison with the cyclostationary detection, but it has the same accuracy of detection. When $P$ is 0.5 , it means half of the channels in the combined energy and cyclostationary detector are concluded at the energy detection stage, and the other half need to pass through cyclostationary detection. The mean detection time of the I3S is still less than that of cyclostationary detection, and has the same accuracy of detection. When $P$ is 0.8 , most of the channels sensed by the combined energy and cyclostationary detector need

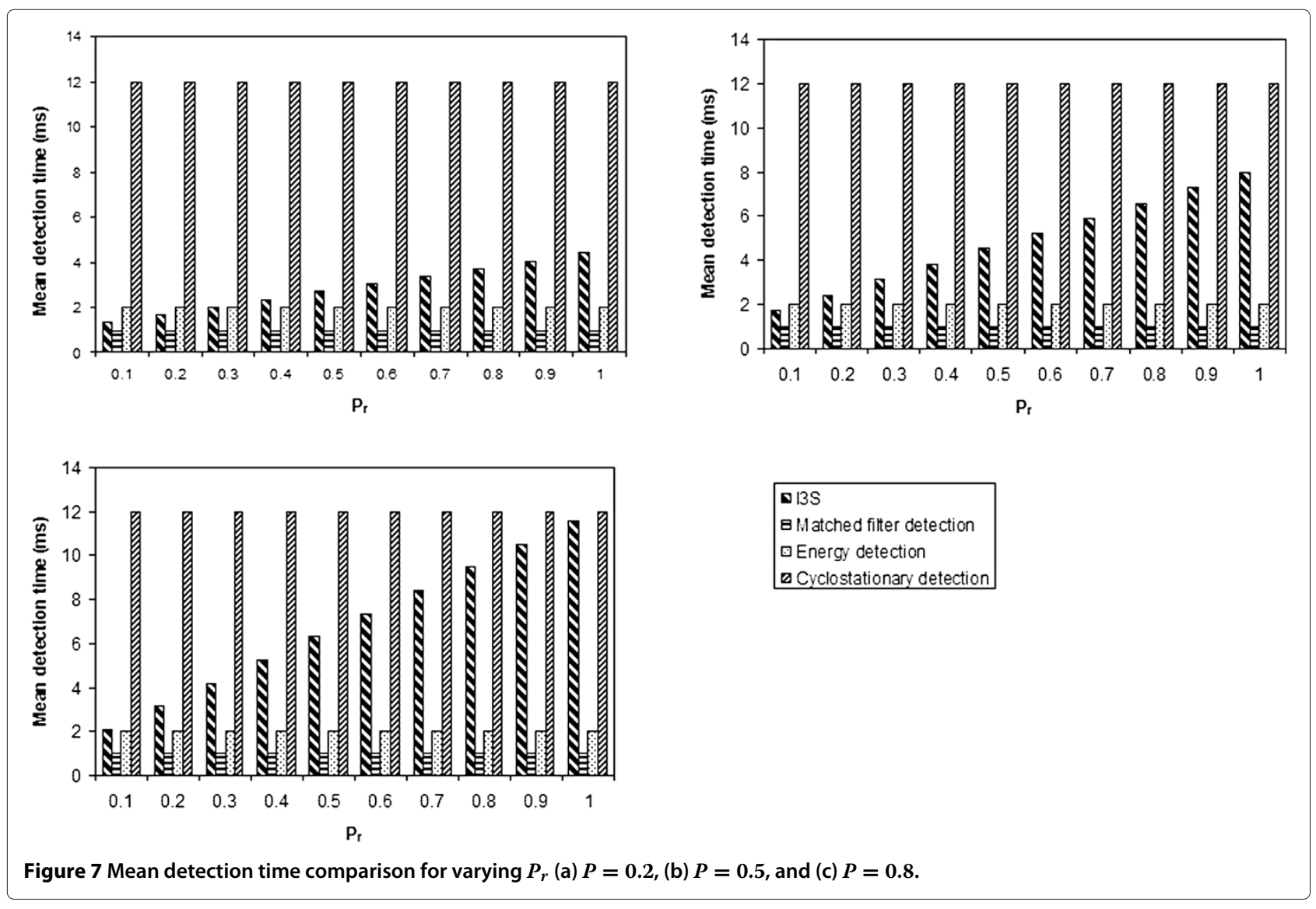


to go through the cyclostationary stage for PU detection, but the accuracy of detection increases. The energy detector is unable to distinguish between the signal energy and noise energy, and is a poor detector under low SNR conditions. Therefore, the accuracy of detection is increased at the cost of mean detection time.

In Table 2, the performance of existing improved local sensing schemes discussed in Section 2 is presented. The two most prominent schemes are the FLD scheme [5] and two-stage spectrum sensing [9], which gives the most reliable results under low SNR conditions. At an average SNR of $-10 \mathrm{~dB}$, the probability of detection of FLD scheme is 0.97 in comparison with 0.99 in case of two-stage spectrum sensing scheme. However, the probability of false alarm for FLD scheme is 0.0001, while the corresponding value is 0.1 in case of two-stage spectrum sensing scheme. Spectrum efficiency can be improved by minimizing false alarms and misdetection causes interference with PU.

It is mentioned in Section 1 that no other scheme considered detection scheme for a geographic region where we have different types of PUs. The prior knowledge of PU waveform is known for some PUs while for the others it is unknown. It is obvious from the results of proposed I3S that the probability of detection is approximately 0.99 for the given probability of false alarm 0.1 at a given average SNR of $-10 \mathrm{~dB}$. According to the draft of IEEE802.22 standard [20], the probability of false alarm should be less than or equal to 0.1 and the probability of detection should be greater than 0.9. The detection time will be much less if the prior information of PU waveform is known. Therefore, the mean detection time depends on the probability that channel would be sensed by combined energy and cyclostationary detector or matched filter.

\section{Table 2 Performance of existing improved spectrum sensing schemes}

\begin{tabular}{|c|c|c|c|}
\hline Spectrum sensing scheme & $\mathrm{SNR}(\mathrm{dB})$ & $P_{f}$ & $P_{d}$ \\
\hline FLD scheme [5] & -10 & 0.0001 & 0.97 \\
\hline $\begin{array}{l}\text { DFT filter bank-based two-stage } \\
\text { spectrum sensing [6] }\end{array}$ & 12 & 0.1 & 0.85 \\
\hline $\begin{array}{l}\text { SNR-based adaptive spectrum } \\
\text { sensing [7] }\end{array}$ & -10 & 0.1 & 0.75 \\
\hline High-speed two-stage detector [8] & -16 & 0.1 & 0.9 \\
\hline $\begin{array}{l}\text { Two-stage spectrum sensing for } \\
\text { CRs [9] }\end{array}$ & -10 & 0.1 & 0.99 \\
\hline $\begin{array}{l}\text { Fast two-stage spectrum detector } \\
\text { for CRs [10] }\end{array}$ & -11 & 0.1 & 0.93 \\
\hline $\begin{array}{l}\text { Combined energy detector and } \\
\text { one order cyclostationary detector } \\
{[11]}\end{array}$ & 10 & 0.1 & 0.94 \\
\hline $\begin{array}{l}\text { A two-stage spectrum sensing } \\
\text { technique for dynamic spectrum } \\
{[12]}\end{array}$ & 6 & 0.1 & 0.99 \\
\hline
\end{tabular}

\section{Conclusions}

In this article, a new local spectrum sensing scheme, namely, I3S, was proposed to improve the utilization efficiency of the radio spectrum by increasing detection reliability and decreasing sensing time. The proposed scheme chooses either the combined energy and cyclostationary detector, or the matched filter detection based on the power and band of interest.

The proposed I3S is compared with the existing transmitter detection techniques. It is concluded that I3S has reliable results with less mean detection time, depending on the prior knowledge of PU waveform. A change in the probability that channels are sensed by combiner energy and cyclostationary detector means that the thresholds of bi-threshold energy detector also change. Therefore, a guideline for network designers to set optimal values of thresholds in bi-threshold energy detector to achieve desired probability of detection is given. The results reveal that for a specific value of average SNR, high probability of false alarm results in a low probability of misdetection because of the decreased threshold. Moreover, it is observed that the I3S provides detection results nearly equal to cyclostationary detection. On the other hand, the mean detection time of the I3S is quite lower than cyclostationary detection in most cases.

\section{Competing interests}

The authors declare that they have no competing interests.

\section{Acknowledgements}

This study was supported by the CITRC (Convergence Information Technology Research Center) support program (NIPA-2012-H0401-12-1003), supervised by the NIPA (National IT Industry Promotion Agency) of the MKE(Ministry of Knowledge Economy), and the Industrial Strategic Technology Development Program (10035610) funded by the MKE. It was partially supported by the Seoul R\&BD Program (SS110012C0214831) and Special Disaster Emergency R\&D Program from National Emergency Management Agency through Kyungil University (2012-NEMA10-002-01010001-2012).

\section{Author details}

${ }^{1}$ Department of Information \& Communication Engineering, Sejong University, Seoul, Republic of Korea. ${ }^{2}$ Sensor System Research Center, Korea Institute of Science and Technology, Seoul, Republic of Korea.

Received: 26 September 2012 Accepted: 22 December 2012

Published: 11 February 2013

\section{References}

1. IF Akyildiz, W-Y Lee, MC Vuran, S Mohanty, NeXt generation/dynamic spectrum access/cognitive radio wireless networks: a survey. Comput. Netw. 50(13), 2127-2159 (2006)

2. TYucek, H Arslan, A survey of spectrum sensing algorithms for cognitive radio applications. Commun. Surv Tutor. 11(1), 116-130 (2009)

3. IF Akildiz, BF Lo, R Balakrishan, Cooperative spectrum sensing in cognitive radio networks: a survey. Phys. Commun. 4(1), 40-62 (2011)

4. A Ghasemi, ES Sousa, Spectrum sensing in cognitive radio networks: requirements, challenges and design trade-offs. IEEE Commun. Mag. 46(4), 32-39 (2008)

5. W Ejaz, NU Hasan, MA Azam, HS Kim, Improved local spectrum sensing for cognitive radio networks. EURASIP J. Adv. Signal Process (2012). http:// asp.eurasipjournals.com/content/2012/1/242 
6. KG Smitha, AP Vinod, PR Nair, in IEEE Proceedings of International Conference on Innovations in Information Technology (IIT). Low power DFT filter bank based two-stage spectrum sensing, (UAE, March 2012), pp. 173-177

7. W Ejaz, NU Hasan, HS Kim, SNR-based adaptive spectrum sensing for cognitive radio networks. Int. J. Innov. Comput. Inf. Control. 8(9), 6095-6106 (2012)

8. S Geethu, GL Narayanan, A novel high speed two stage detector for spectrum sensing. Elsevier Procedia Technol. 6, 682-689 (2012)

9. S Maleki, A Pandharipande, G Leus, in IEEE Proceedings of International Conference on Acoustic Speech and Signal Processing. Two-stage spectrum sensing for cognitive radios, (USA, March 2010), pp. 2946-2949

10. PR Nair, AP Vinod, KG Smitha, AK Krishna, Fast two-stage spectrum detector for cognitive radios in uncertain noise channels. IET Commun. 6(11), 1341-1348 (2012)

11. W Yue, B Zheng, Q Meng, W Yue, Combined energy detection one-order cyclostationary feature detection techniques in cognitive radio systems. J China Univ. Posts Telecommun. 17(4), 18-25 (2010)

12. L Luo, NM Neihart, S Roy, DJ Allstot, A two-stage sensing technique for dynamic spectrum access. IEEE Trans. Wirel. Commun. 8(6), 3028-3037 (2009)

13. H Urkowitz, Energy detection of unknown deterministic signals. Proc. IEEE. 55(4), 523-531 (1967)

14. A Fehske, JD Gaeddert, JH Reed, in IEEE Proceedings of Dynamic Spectrum Access Networks (DySPAN). A new approach to signal classification using spectral correlation and neural networks, (USA, November 2005), pp. $144-150$

15. D Cabric, SM Mishra, RW Brodersen, in Proceedings of 38th Asilomar Conference on Signals, Systems and Computers (Implementation issues in spectrum sensing for cognitive radios, (USA, November 2004), pp. $772-776$

16. S-Y Chang, Analysis of Proposed sensing schemes. IEEE P802.22 Wireless RANs (2006)

17. W Yue, B Zheng, Spectrum sensing algorithms for primary detection based on reliability in cognitive radio systems. J. Comput. Electr. Eng. 36(3), 469-479 (2010)

18. A Sahai, N Hoven, R Tandra, in Proceedings of Allerton Conference on Communication, Control, and Computing. Some fundamental limits in cognitive radio, (USA, October 2004), pp. 1662-1671

19. D Bhargavi, CR Murthy, in IEEE Proceedings of International Workshop on Signal Processing Advances in Wireless Communications (SPAWC). Performance comparison of energy, matched-filter and cyclostationarity-based spectrum sensing, (Morocco, June 2010), pp. 1-5

20. IEEE Computer Society, IEEE Std 802.22-2011 Part 22: Cognitive Wireless RAN Medium Access Control (MAC) and Physical Layer (PHY) Specifications: Policies and Procedures for Operation in the TV Bands. IEEE Standard for Information Technology , 1-672 (2011)

doi:10.1186/1687-1499-2013-26

Cite this article as: Ejaz et al:: 13S: Intelligent spectrum sensing scheme for cognitive radio networks. EURASIP Journal on Wireless Communications and Networking 2013 2013:26.

\section{Submit your manuscript to a SpringerOpen ${ }^{\circ}$ journal and benefit from:}

- Convenient online submission

- Rigorous peer review

- Immediate publication on acceptance

- Open access: articles freely available online

- High visibility within the field

- Retaining the copyright to your article

Submit your next manuscript at $\boldsymbol{\wedge}$ springeropen.com 